\title{
CORRUPCIÓN PRIVADA: \\ MÁS SOMBRAS QUE LUCES EN EL CASO HONDUREÑO \\ Y LA COMPARACIÓN CON NORMATIVAS INTERNACIONALES
}

\section{Juan Carlos Aguilar ${ }^{1}$}

DOI: https://doi.org/10.5377//rd.v41i1.10498

\section{RESUMEN:}

La presente investigación esta orientada a mostrar la significancia e importancia que conlleva el tipificar como delito a la corrupción privada, en el sentido de que, a la luz de los tratados internaciones, la diferente normativa regional como la europea y los autores que evalúan el impacto que genera este flagelo no solo en la esfera pública, sino también en el ámbito privado. Se procedió a realizar la ponderación entre los intereses jurídicos que dicho delito debe proteger. Asimismo, la percepción que tiene la ciudadanía hondureña con respecto al delito de corrupción privada, con relación a introducir el tema en la dinámica hondureña. Posteriormente se realiza un análisis del deber ser para el caso hondureño y como ayudará al país la tipificación del delito en nuestra normativa interna.

\section{PALABRAS CLAVE:}

Corrupción Privada, Corrupción Activa, Corrupción Pasiva, Interés Jurídico y Deber de Fidelidad.

Fecha de recepción: 31 de agosto de 2020 Fecha de aprobación:10 de noviembre de 2020

\footnotetext{
1 Abogado por la Universidad Nacional Autónoma de Honduras y Máster en Estrategias Anticorrupción y Políticas de
} Integridad por la Universidad de Salamanca, España. Correo electrónico: jcaguilarm911@gmail.com 


\title{
PRIVATE CORRUPTION: \\ MORE SHADOWS THAN LIGHTS IN THE HONDURAN CASE \\ AND THE COMPARISON WITH INTERNATIONAL REGULATIONS
}

\section{Juan Carlos Aguilar ${ }^{2}$}

DOI: https://doi.org/10.5377//rd.v41i1.10498

\begin{abstract}
:
This investigation is aimed at showing the significance and importance of criminality of private corruption, in the sense that, in the light of international treaties, different regional legislation such as European and authors that assess the impact of this scourge not only on the public sphere, but also on the private sphere. The weighting of the legal interests to be protected by that offence was carriedout. Likes, Honduran citizens' perception of the crime of private corruption. Subsequently, an analysis of the duty to be carried out for the Honduran case and how the country would help the criminalizement in our internal legislation.
\end{abstract}

\section{KEYWORDS:}

Private Corruption, Active Corruption, Passive Corruption, Legal Interest and Duty of Fidelity.

Date received: August 31, 2020

Approval date: November 10, 2020

2 Lawyer from the Universidad Nacional Autónoma de Honduras and Master in Anti-Corruption Strategies and Integrity Policies from the University of Salamanca, Spain. Email: jcaguilarm911@gmail.com 


\section{INTRODUCCIÓN}

El fenómeno de la corrupción es tan antiguo como la existencia misma de la humanidad. Pero a raíz del creciente impacto generado y las consecuencias nefastas que trae consigo, fue necesario ponerle atención especializada. Ello se da hace unas 3 o 4 décadas atrás, cuando surge la preocupación a nivel mundial por la problemática que trae consigo la corrupción, y, por lo que fue catalogada como un problema de índole global, ya que si bien es cierto la afección es generada en mayor o menor medida, pero todos los países estaban siendo permeados por la misma.

En ese sentido, por los muchos escándalos de corrupción que salieron a la luz en esos años en las principales naciones del mundo, se comenzó a conformar grupos de coalición para ponerle un freno a dicho flagelo; a pesar de ello algunos de los países más importante comenzaron por su cuenta a tipificar conductas que consideraban constitutivas de corrupción. Pero fue hasta finales del siglo pasado e inicios del presente cuando existió el afán de organizarse para crear mecanismos internacionales que tipificaran como corrupción las conductas típicas que conllevaban acciones de índole antijurídicas.

Debido a lo anterior es que surge el primer instrumento internacional en combate a la corrupción; la Convención Interamericana Contra la Corrupción, que a pesar de ser muy completo con relación a la corrupción pública descuido otros campos de acción como la corrupción privada. Por ende, se da la necesidad de crear el mecanismo internacional por excelencia, en cuanto al combate a la corrupción y es la Convención de Naciones Unidad Contra la Corrupción o la también conocida como Convención de Mérida ${ }^{3}$, que por su carácter universal es el instrumento que más países han suscrito y puesto en marcha para lograr la tipificación de las conductas establecidas en el mismo cuerpo legal y que constituyen corrupción.

Asimismo, dicho instrumento si contempla las conductas que constituyen la corrupción privada. Pero también se refleja la incapacidad de muchos de los Estados que no han tipificado dicho delito o han tardado muchos años en hacerlo (caso Honduras).

Por lo tanto, la finalidad del presente artículo se orienta en mostrar los problemas que trae consigo dejar al descubierto un campo de acción tan grande como lo es el sector privado. En ese mismo orden de ideas es de vital importancia evidenciar las ventajas y utilidades que supone el generar una protección por parte del Estado para este ámbito en el que también se constituye la corrupción y no solo en el sector público propiamente dicho, sino que este ámbito privado que también se ve inmiscuido en la corrupción generada desde la esfera publica, es decir la corrupción común y corriente que conocemos cada uno de nosotros.

\section{METODOLOGÍA}

La presente investigación ha sido desarrollada a través de una metodología descriptiva y explicativa, ello en razón, de

3 Véase el preámbulo en Centro Internacional de Viena Convención de Naciones Unidas Contra la Corrupción (2004). New York. 
que los mecanismos utilizados se basan en legislaciones, instrumentos internacionales y doctrina de diversos autores. Con la finalidad de lograr mediante el estudio de la parte teórica, para así plasmar los avances desde el punto de vista formal del derecho, haciendo a su vez comparaciones entre las modalidades de corrupción. Para posteriormente y partiendo de ello realizar los respectivos análisis a los puntos torales en la investigación, que van desde establecer una definición clara y aceptada universalmente de la corrupción privada hasta observar cuál ha sido la evolución que ha tenido la misma desde el punto de vista del interés jurídico protegido que se ha visto armonizado por la mayoría de los países en cuestión.

\section{LA CORRUPCIÓN PRIVADA Y SUS MODALIDADES}

El combate a la corrupción ha sido una de las misiones comunes en el reciente mundo globalizado; esto debido a que el flagelo ha permeado todas las esferas de las sociedades más desarrolladas y aún más fuerte en las sociedades en vías de desarrollo. Por lo que se ha decidido hacerle frente de una manera directa a través de políticas orientadas desde un punto de vista represivo y que se considera que allí es donde se comete el principal error en esta lucha. Por otro lado, los recursos financieros y humanos se han destinado para combatir a la corrupción en el sector público, que si bien es cierto es la más conocida por ser la que más atención ha generado. Dejando a un lado a otros campos donde la corrupción permea y afecta directamente a los ciudadanos que son los que sufren las consecuencias más nefastas que se propician de esta. En ese sentido es oportuno evidenciar la importancia de conocer y tratar a la corrupción privada o también conocida como corrupción en los negocios.

Antes de comenzar a abordar el tema de la corrupción privada propiamente dicha, es oportuno establecer un concepto de corrupción general a fin de generar una perspectiva más amplia con respecto a este flagelo. Debido a ello es que los autores establecen que la corrupción es "toda violación por parte de un individuo dotado de poderes de decisión de las reglas que rigen la actividad del agente, con el objetivo de procurar para sí mismo o para un tercero una ventaja de cualquier índole" ${ }^{\text {. En }}$ ese sentido un concepto general de lo que es corrupción se puede moldear tanto a la esfera pública como privada, por ello, es necesario entender que de la precitada definición se desprende la actividad o teoría del agente y principal. Y debemos comprender esta teoría como aquella protagonizada por el principal (funcionario público o un alto directivo de una empresa privada) que en su condición de superior jerárquico y con suficiente poder en cuanto al manejo de la entidad pública u organización privada, decide revestir de cierto poder en la toma de decisiones al agente (empleado de la entidad pública o de la organización privada) para que este represente los intereses que han sido otorgados por el principal. En otras palabras lo importante es entender la relación de confianza que se rige entre el principal y agente, ello debido a que en el caso de que exista el quebrantamiento de esa relación de confianza, el perjudicado

4. Caruso, F. (2009). El Concepto de Corrupción. Su Evolución Hacia un Nuevo Delito de Fraude en el Deporte Como Forma de Corrupción en el Sector Privado. Revista De Derecho, (6ta), 152 . 
directamente será la administración pública o la empresa privada, en razón de que sus intereses no estarán siendo protegidos de la mejor manera, o se han dejado a un lado, debido a que el agente ha decidido velar por intereses particulares o de terceros ajenos a la organización.

Estableciendo una vez el concepto de corrupción general es momento de abordar directamente a la corrupción privada, que debemos entenderla como la protagonizada por agentes económicos que, mediante comisiones, sobornan o intentan sobornar a los responsables de entidades privadas para lograr la adjudicación de contratos; estableciendo así relaciones ilícitas privilegiadas, todo ello con finalidad de hacer o dejar de hacer en beneficio del que soborna ${ }^{5}$. En ese sentido, la pieza clave en la corrupción privada es el soborno realizado por un particular que generalmente representa a otra entidad privada o que actúa como autónomo, ello con la finalidad de obtener esa ventaja ilícita por sobre las demás empresas participantes y así adjudicarse el negocio jurídico que este en juego. Ahora bien, la tarea desempeñada por la persona que recibe el soborno es la de hacer que se esa ventaja se materialice o bien dejar de hacer algo para que el resultado sea el mismo. Cuando se da la existencia de corrupción privada también existe el quebrantamiento a la teoría del agente y principal, debido a que efectivamente se vulnero esa relación de confianza que se maneja bajo el deber de fidelidad y que por lo tanto ese empleado conocido como agente dejo de salvaguardar los intereses de la empresa privada que le fueron confiados por el principal.

5 Borrallo, A.(2015). Los Delitos de Corrupción en los Negocios tras la reforma Penal de 2015: Bases Político-Criminales y Técnico Jurídicas (1era ed., p. 53). Madrid: Editorial de Madrid.
De la corrupción privada se generan dos modalidades de corrupción y son la corrupción privada activa y la corrupción privada pasiva. Antes de definir las modalidades tanto activa como pasiva es importante hacer la analogía con lo que conocemos como cohecho activo y cohecho pasivo, el cual se encarga de regular y castigar comportamientos corruptos desde la esfera pública. Logrando así las modalidades de corrupción ocupar esos espacios vacíos y descuidados en el sector privado.

Por lo tanto, debemos entender a la corrupción privada activa a la luz de lo planteado por la Decisión Marco relativa a la lucha Contra la Corrupción en el Sector Privado como "el prometer, ofrecer o entregar, directamente o a través de un intermediario, a una persona que desempeñe funciones directivas o laborales para una entidad del sector privado, una ventaja indebida de cualquier naturaleza para dicha persona o para un tercero, para que esta realice o se abstenga de realizar un acto incumpliendo sus obligaciones". A su vez la Decisión Marco estableció para la corrupción privada pasiva lo siguiente "pedir o recibir, directamente o a través de un intermediario..." "6

A fin de no ser repetitivo entre la definición de una y otra considerando que el concepto es el mismo y lo que cambia en cada una de ellas es la acción de los involucrados, por lo tanto, debe quedar claro la diferencia sustancial entre la modalidad activa y pasiva. En que en la activa quien ejecuta la acción de entablar la relación mediante el soborno es el particular mediante la promesa, el ofrecimiento o la

6 Diario Oficial de la Unión Europea. Decisión Marco 2003/568/JAI del Consejo (2003). Bruselas. 
entrega del soborno. En contraposición tenemos a la modalidad pasiva, en donde quien cuenta con el ánimo de generar esa relación es el empleado de la empresa quien solicita o recibe el soborno por parte del particular. Otro aspecto importante es que el soborno se puede materializar desde el punto de vista de dinero, bienes, viajes y hasta favores de índole sexual, por lo que un ejemplo muy común en el caso de la corrupción privada es que los sobornos se materializan mediante el pago de viajes a congresos organizados en otros países que son patrocinados por las farmacéuticas para los doctores. De esa forma dichas farmacéuticas se aseguran de que estos patrocinen y utilicen los medicamentos de dicha casa; dejando en posición de desventaja a las demás farmacéuticas que en algunas ocasiones cuentan con un mejor producto hasta por menos precio, pero que por motivos de la ventaja ilícita otorgada mediante el soborno no son las elegidas para su utilización.

\section{INTERÉS JURÍDICO PROTEGIDO EN EL CASO DE LA CORRUPCIÓN PRIVADA}

Con respecto al interés jurídico protegido en esta modalidad de corrupción, se ha generado debate sobretodo a nivel europeo, debido a que es el espacio geográfico que mayormente tiene tipificado como delito este tipo de conductas. Para el caso del presente artículo abordaremos de manera concreta los casos de legislaciones como la italiana, alemana y española. Donde se muestra una evolución de los intereses jurídico y de cómo se ha logrado cierta armonización legislativa para poder tipificarlos. Asimismo, se hará énfasis en el modelo que mejor se adapta a las realidades y escenarios jurídicos de cada país.

\section{CASO ITALIANO}

En el caso italiano hasta antes del año 2017, el interés jurídico era de índole -patrimonialdebido a que, para la existencia del delito de corrupción privada se tendría que vulnerar directamente el patrimonio de las empresas involucradas. En ese sentido podría darse el caso de que se produzcan dicho perjuicio como consecuencia de un aumento en el precio de una determinada operación comercial, después, de que una empresa le pague un soborno a otra empresa distinta ${ }^{7}$. Posteriormente, se decidió suprimir ese componente patrimonialista del delito, introduciendo así el deber de fidelidad inherente al cargo de los empleados y directivos.

Debiendo entender al deber de fidelidad como aquella relación de confianza donde se le otorga la protección de intereses al empleado para que este represente a la empresa, es decir, si existe un quebrantamiento al deber de fidelidad lo que ocasiona el mismo es esa perdida de confianza y por ende la vulneración de los intereses en la sociedad comercial. Además, es importante resaltar que la modalidad de corrupción privada en el caso italiano no es de acción pública, es decir, que el delito no se persigue de oficio, sino a iniciativa de parte y es aquí donde se encuentra uno de los principales obstáculos para detener este tipo de corrupción; debido a que las empresas optan por implementar acciones disuasivas internas con el ánimo

7 Martín, N. (2002). La corrupción en el sector privado. Revista Penal, (10), 67. 
de que la imagen de la empresa no quede manchada a nivel de medios de comunicación y que por ende su reputación se coloque en un lista de empresas con acciones corruptas realizadas por los mismos empleados, lo que evidentemente generaría un detrimento para su desarrollo comercial.

\section{CASO ALEMÁN}

Ahorabien, el casoalemán es muy particular, debido a que fue uno de los primeros países que logro tipificar como delito a la corrupción privada, pero su protección se basaba en la -competencia-. Por lo que, ese delito estriba en la salvaguarda de la competencia empresarial o profesional de la contratación de negocios y por ende se asume que se están protegiendo interés de carácter colectivos, causando afección al normal funcionamiento del mercado ${ }^{8}$. La particularidad de este modelo alemán a diferencia por ejemplo del italiano es que su protección era más completa e integral, ya que no se limitaba solamente a proteger a las empresas participantes en la relación ilícita, sino más bien su protección se extendía a todas las empresas que se veían afectadas como un efecto domino producto de las relaciones ilícitas entabladas.

Posteriormente se realizada la modificación a la Gesetz zur Bekämpfung der Korruption o la Ley de Lucha Contra la Corrupción en español, donde se originó una ampliación del modelo de competencia que se complemento con el deber de fidelidad. Logrando así un mejor y más completo modelo de protección a los intereses jurídicos, debido a que la

8 Martín, N., \& Tiedemann, K. (2004). Protección penal de la competencia (1er ed., p. 91). Valencia: Editorial valenciana. protección se da a nivel macro y micro; en razón de que la protección a los intereses colectivos esta presente y por otro se da una especializada atención a las empresas involucradas directamente, por lo que el modelo mixto es el más adecuado.

\section{CASO ESPAÑOL}

Y finalmente, el caso español que la tipificación del delito fue tardía, ya que fue hasta el año 2010 cuando introdujeron a su Código Penal el delito bajo la modalidad mixta alemana, pero fue en el año 2015 cuando se realizó una reforma donde se dejo solamente el deber de fidelidad como interés jurídico protegido. Con la particularidad que esa misma reforma España implementa un subtipo de corrupción privada la llamada -corrupción en los deportes- al igual que en Honduras en el nuevo Código Penal en el artículo 419. Con la misma dinámica que la corrupción privada, pero su orientación esta apegada al mundo de los deportes, un ejemplo claro y conocido de este tipo de corrupción es lo que ocurrió en Italia entre 2004 y 2006 con el escandalo de corrupción deportiva denominado como calciopolis ${ }^{9}$, donde se pagaron sobornos por parte de los directivos a los árbitros para favorecer a ciertos equipos. Beneficios que se materializaban desde el campo de juego hasta altos intereses económicos para las cúpulas corruptas que englobaban a jugadores, dirigentes y árbitros.

\section{CORRUPCIÓN PRIVADA PARA EL}

9 Run, E., AmericanoNHLPokerPoloRugbyesportsXFL, \&., Motor, D., Games, X., \& Americano, F. (2020). DE PALMA: "Calciópolis", un caos. Retrieved 9 November 2020, from https://www.espn.com.co/noticias/nota?id $=442102$ 


\section{CASO HONDUREÑO}

Honduras fue de los primeros países en suscribir y ratificar la Convención de Naciones Unidas Contra la Corrupción o Convención de Mérida $^{10}$. A pesar de los esfuerzos realizados para tipificar más no combatir las conductas de corrupción pública que han sido adaptadas en el Código Penal hondureño. Aún queda a deber el Estado de Honduras, debido a que las conductas establecidas en el artículo 21 de la Convención son las constitutivas de corrupción privada activa y pasiva. Pero que, hasta la fecha, casi 20 años después apenas se comienza a dar los primeros pasos, lo que es positivo, pero a la vez muy tardío. En ese sentido, el capitulo III del nuevo Código Penal versa sobre los delitos de corrupción en los negocios entre particulares o mejor conocidos como corrupción privada, en donde el artículo 418 habla someramente sobre la corrupción activa y pasiva pero sin llegar a profundizar en estas conductas constitutivas de un ilícito penal. Asimismo, las penas establecidas en la legislación hondureña se orientan a penas privativas de la libertad, inhabilitaciones y multas al efecto, lo que en ese sentido si es considerado integral el castigo que deben recibir las personas que delincan bajo esa modalidad; por otro lado el interés protegido en apego a lo establecido expresamente en la legislación es orientado a lo que se aplico en el modelo alemán al inicio cuando regulaban la protección exclusiva a la competencia. Considerando que el sector privado en el país esta involucrado en acciones de corrupción no solo en la esfera privada, sino también en la pública.

10 Centro Internacional de Viena. Convención de Naciones Unidas Contra la Corrupción (2004). New York.
En ese sentido, diversos autores expresan que "las conductas de corrupción en el sector privado son aún -lícitas- en buena parte del mundo occidental" ${ }^{11}$. Esto se da debido a que en Latinoamérica se ha descuidado en buena parte el combate a la corrupción privada. Y en el caso de Estados Unidos que a mediados de los 70's debido al escandalo de watergate, donde a través de una investigación realizada por el Congreso de los Estados Unidos y posteriormente por la Comisión Controladora de Acciones y Valores, se logro establecer que más de 300 empresas privadas sobornaban a los funcionarios estadounidenses ${ }^{12}$. Sin embargo, ese precedente de soborno transnacional sirvió para la creación del Foreign Corrupt Practices Act el cual serviría para regular la corrupción entre las empresas privadas.

Por lo tanto, y a luz del estudio realizado por el Sondeo de Opinión Pública, en su decima edición del año 2020 denominada "Percepciones sobre la situación hondureña en el año 2020". Se hacer ver cuál es la percepción de la ciudadanía hondureña con respecto a la corrupción en la empresa privada. Obteniendo como resultado que el $63.9 \%$ de los ciudadanos considera que la corrupción esta muy extendida en la empresa privada $^{13}$; estableciendo de esa forma lo

11 Cerina, G. (2011). La comunidad internacional y la lucha contra la corrupción en el sector privado. Revista de Derecho Penal, (7), 11.

12 Manfroni, C. (1998). Soborno transnacional. Il Diritto Penale, (1), 29.

$13 \mathrm{Al}$ respecto, véase: Equipo de Reflexión, Investigación y Comunicación de la Compañía de Jesús (ERIC). (2020). Sondeo de Opinión Pública Décima Edición (p.3). El Progreso, Honduras. Al respecto, véase: Equipo de Reflexión, Investigación y Comunicación de la Compañía de Jesús (ERIC). (2020). Sondeo de Opinión Pública Décima Edición (p.3). El Progreso, Honduras. 
que se mencionó supra con respecto a la participación de las empresas privadas en las acciones constitutivas de corrupción.

A sabiendas de que esta percepción esta catalogada por la sensación de lo que se sabe de la participación de las empresas en corrupción pública, sin embargo, si se conociera lo que ocasiona la corrupción privada a la justa y leal competencia, al daño al mercado y al Estado de Derecho ese indicador estaría por encima de ese casi $64 \%$.

Siendo ese uno de los principales daños que ocasiona la corrupción privada, un daño al mercado y por ende es el Estado el garante de establecer medidas que le pongan un freno a este tipo de conductas, debido a que un mercado debe autorregularse según lo establecido inicialmente por el Estado. Pero lo que, sucede eso cuando este mercado es incapaz de lograrlo porque se ha salido de control y se han establecido monopolios o duopolios debido a la falta de igualdad en las relaciones comerciales, es allí donde entra en escena el garante de tutelar los derechos mediante la implementación de políticas y medidas que vengan a darle esa libre y leal competencia a cada una de las empresas que participan en un determinado rubro que componen un mercado. Por lo tanto, un Estado como el hondureño debe de implementar las medidas que sirvan para frenar este tipo de conductas, que sin lugar a dudas suceden en Honduras, primero mediante la tipificación de la conducta ilícita de una manera más integral, segundo estableciendo las normas mediante las cuales se determinen las reglas a seguir, así como los mecanismos y procedimientos de denuncia a fin de contener esas acciones.
Y finalmente, pero no menos importante y que en realidad debería de ser lo primordial en todas las esferas a fin de tratar de controlar los índices de corrupción, es la prevención generalizada; que se logra en esencia con una formación integral, es decir, dentro de la educación y de los valores enseñados a los niños, jóvenes y posteriormente adultos, de esa manera inculcar políticas anticorrupción mediante formación en transparencia, denuncia, integridad en la administración pública y sobre todo las consecuencias nefastas que trae consigo la corrupción, que no solo permea en ámbito donde se realiza, sino que su efecto dominó genera un impacto tan grande que sus efectos a nivel macro son los que tienen a gran parte de Latinoamérica en vías de desarrollo, con educación, salud, altos niveles de desempleo, órganos de justicia ineficaces entre muchos otros factores que funcionan de manera precaria.

Finalmente, en Honduras es necesaria la implementación verdadera de un modelo anticorrupción que cubra en especifico al sector privado; que sea realizado desde un punto de vista amplio para que de esa manera satisfaga de forma eficiente su campo de acción. De esa forma instaurar un modelo mixto como lo desarrollamos supra donde la protección este orientada a todos los participantes en las relaciones jurídico comerciales y también se tenga una protección especializada en las empresas de donde se tiene su origen la conducta ilícita, bien sea para identificar y sancionar de una forma eficaz o para que sirva de una manera que atenué la responsabilidad, ya que, no en todas las ocasiones las empresas son las responsables por realizar este tipo de acciones, sino son los empleados que se 
aprovechan de la empresa para beneficiarse de esta. En ese sentido la responsabilidad debe ser excluyente, es decir, que tanto el empleado como la empresa debe de responder separadamente por sus acciones expresamente y no ser responsable ambos por la condena de alguno de ellos.

\section{CONCLUSIONES}

El Estado de Honduras a quedado a deber con relación a la tipificación de un delito que regule la corrupción privada de una manera integral, ya que, ha tenido todos los mecanismos necesarios para realizarlo, mediante la suscripción de convenciones, contando además con el problema latente de la corrupción en el sector privado, pero aún así se ha tardado en implementarlas y cuando lo hizo fue de manera incompleta.

Observamos como el combate a la corrupción debe ser integral, de manera que no solo hay que centrarse en políticas represivas, sino más bien en las preventivas; orientando esas políticas represivas a situaciones donde verdaderamente se desincentive al delincuente de cometer actos constitutivos de corrupción, debido a que, de no lograr ese desincentivo de las conductas delincuenciales, al individuo no le importará por muy alta que sea la medida represiva pero que en realidad no es eficaz.

Asimismo, determinamos como los modelos correspondientes a los intereses jurídicos han ido evolucionando y se han armonizado hasta cierto punto en la normativa europea. Para el caso hondureño lo ideal sería replicar el modelo más completo y que mejor se adpate a las necesidades del país.
Se necesitan más mecanismos de control, supervisión, seguimiento y veeduría a las políticas anticorrupción, ello con el afán de dotar de mayor transparencia y fortalecer el sistema. Otorgándole de esa forma la apertura a sectores del país que sirvan como fiscalizadores del Estado mismo. Logrando de esta forma fortalecer el Estado de Derecho con instituciones más solidas, con redición de cuentas y que estén debidamente supervisadas en la implementación de sus tareas.

\section{BIBLIOGRAFÍA}

\section{Legislación}

Diario Oficial de la Unión Europea. Decisión Marco 2003/568/JAI del Consejo (2003). Bruselas.

Centro Internacional de Viena. Convención de Naciones Unidas Contra la Corrupción (2004). New York.

\section{Artículos de revistas}

Borrallo, A. (2015). Los Delitos de Corrupción en los Negocios tras la reforma Penal de 2015: Bases Político-Criminales y Técnico Jurídicas (1era ed., p. 53). Madrid: Editorial de Madrid.

Caruso, F. (2009). El Concepto de Corrupción. Su Evolución Hacia un Nuevo Delito de Fraude en el Deporte Como Forma de Corrupción en el Sector Privado. Revista De Derecho, (6ta), 152.

Cerina, G. (2011). La comunidad internacional y la lucha contra la corrupción en el sector privado. Revista de Derecho Penal, (7), 11. 
Manfroni, C. (1998). Soborno transnacional.

Il Diritto Penale, (1), 29.

Martín, N. (2002). La corrupción en el sector privado. Revista Penal, (10), 67.

Martín, N., \& Tiedemann, K. (2004). Protección penal de la competencia (1er ed., p. 91). Valencia: Editorial valenciana.

Estudios de campo

Equipo de Reflexión, Investigación y Comunicación de la Compañía de Jesús (ERIC). (2020). Sondeo de Opinión Pública Décima Edición (p.3). El Progreso, Honduras.

Web

Run, E., AmericanoNHLPokerPoloRugbyesportsXFL, \&., Motor, D., Games, X., \& Americano, F. (2020). DE PALMA: "Calciópolis", un caos. Retrieved 9 November 2020, from https://www.espn.com.co/noticias $/$ nota? id $=442102$ 